\title{
Polishing characteristics of sensitive glasses - experimental investigation
}

\author{
Christian Vogt and Rolf Rascher \\ University of Applied Sciences, 94469 Deggendorf, Germany
}

\begin{abstract}
Polishing is according to the current state of the art, one of the key processes in precision optics. Many specialists deal with the most diverse applications of these technologies and develop stable, reproducible techniques. In this article we present results for the investigation of different effects in the initial polishing phase in combination with different materials.
\end{abstract}

Computer Controlled Polishing (CCP) [1] is state of the art when it comes to fine and ultrafine processing of optical glasses. These sub-aperture methods are based on the principle of feed-controlled removal, i.e. in areas of the sample with a high excess of material, polishing takes place for longer, i.e. it lasts longer, then in other areas. The accuracy of the processes therefore depends on how stable the removal rate is over time. Methods with a high removal rate usually show a higher absolute variance than those with a low removal rate. Accordingly, they are used sequentially and specified with different, achievable accuracies.

For example, polishing with ball tools with polishing foils is usually found in the pre-processing stage, where several micrometers of height of arrow are removed within a few minutes (depending on material and dimension). The achievable form deviations are usually in the range of several $100 \mathrm{~nm}$.

In order to achieve even higher accuracies, processes follow, e.g. with bonnet or fluid jet tools, which enable more precise work due to lower relative speeds and pressures.

This is followed by processes such as MRF or IBF for the high-precision sector.

To make the entire production chain more efficient, it is worth taking a closer look at the foil-based pre-machining processes. While standard glasses, such as quartz or BK7 generally do not cause any or only minor problems, the situation is quite different for very soft glasses with hardness below 400 Knoop.
Particularly when polishing new tools, there are large fluctuations in the removal rate, which we are currently investigating more closely with the aid of a specially developed laboratory test rig.

In a first step the polishing behavior of new tools was investigated. Windows with $12 \times 16 \mathrm{~mm}$ size were polished on different glasses (table 1).

Figures 1-2 clearly show the fluctuations that occur during the initial polishing phase. Figures 2-4 show a more stable performance after initial wear has occurred. All glasses were polished under identical conditions. SF57 showed the largest fluctuations of all glasses during initial polishing. A separate polishing before the actual processing is definitely reasonable here, as it is also practiced by experienced fine opticians.

The standard in the industry is often to bring newly installed polishing processes directly into production. In general, it is unknown how long a certain constellation of polishing processes needs until stable results are delivered. In the presented project, we have experimentally determined the volume needed for polishing, depending on the glass. Based on these results, scrap can be reduced in industrial production, which is currently under investigation.

We are very grateful to Dr. Oliver Faehnle for valuable discussions and essential thoughts on this topic.

* Corresponding author: christian.vogt $@$ th-deg.de 
Polishing pad material:

Tool main body

Elastic layer

Parameters

Spotdiameter

Glas materials
Polyurethane, Zirconia filled

Density (lb/ft3): $34-42$

Hardness: 33-43

3D-printed, PLA, diameter $57 \mathrm{~mm}$

3D-printed polyurethane, shore-hardness ca. $75,70 \%$ filling

$6.4 \mathrm{~N}$ tool load, tool speed $2000 \mathrm{rpm}$, feed speed $20 \mathrm{~mm} / \mathrm{min}$

$5 \mathrm{~mm}$

N-LAF21, N-SF57, SF57

Table 1. Process information
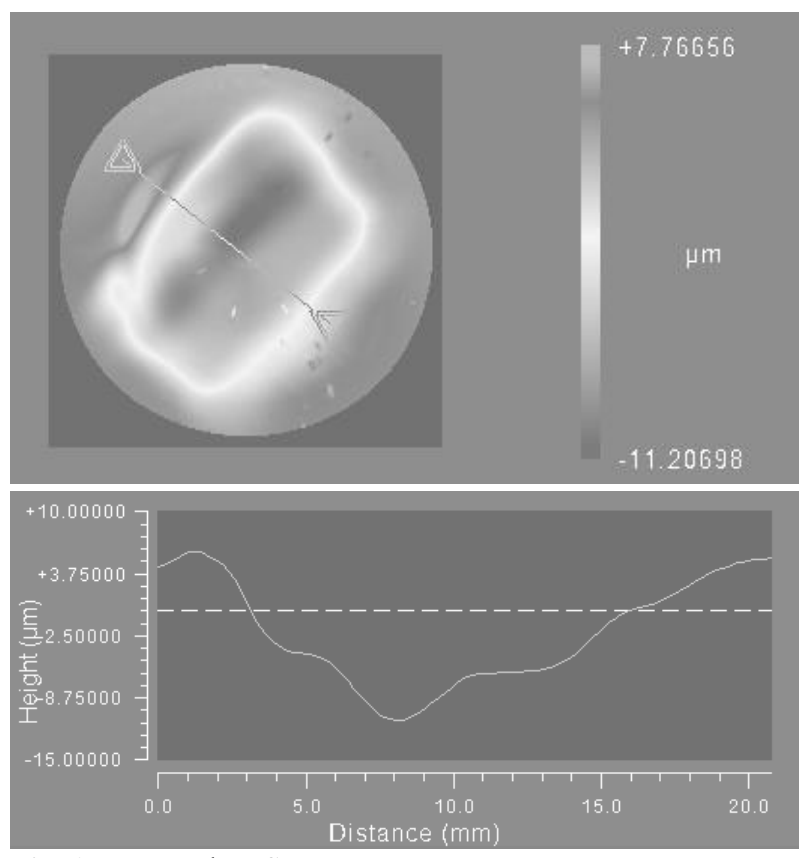

Fig. 1. New tool on SF57

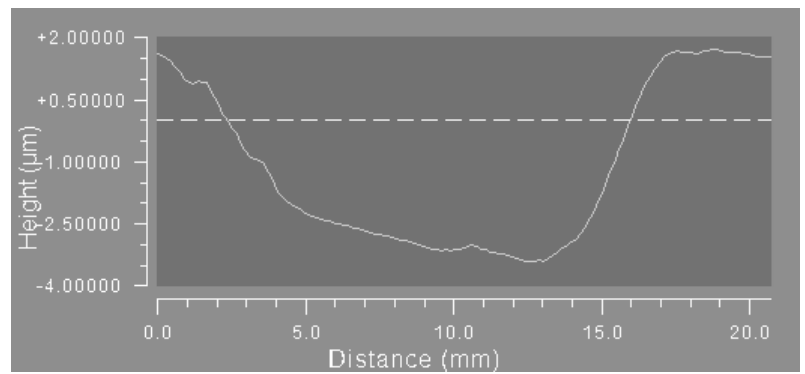

Fig. 3. Slightly worn tool on N-SF57

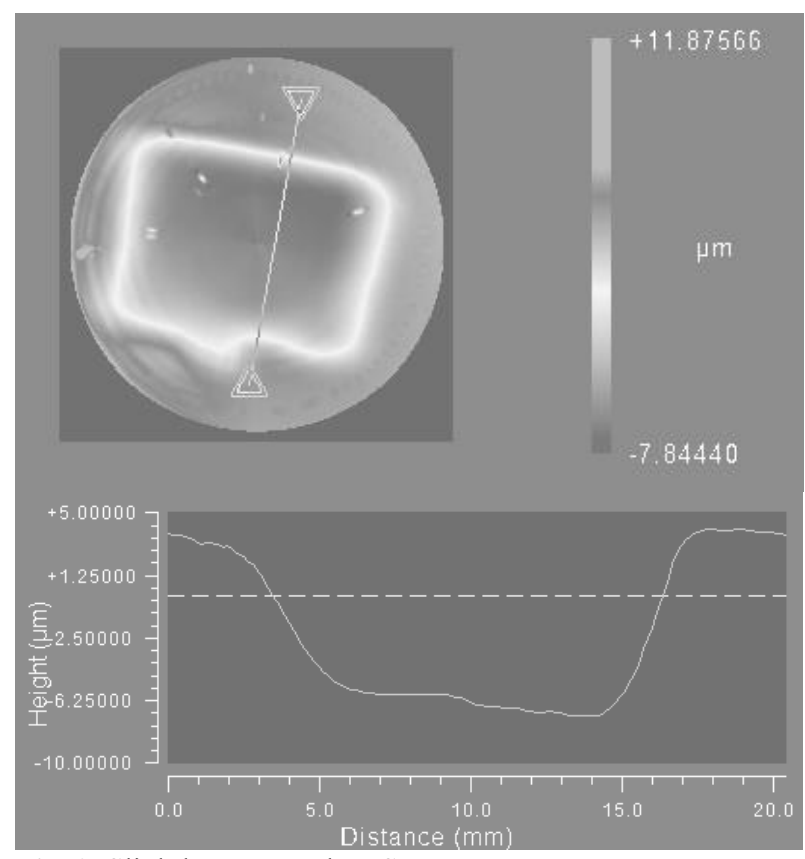

Fig. 2. Slightly worn tool on SF57

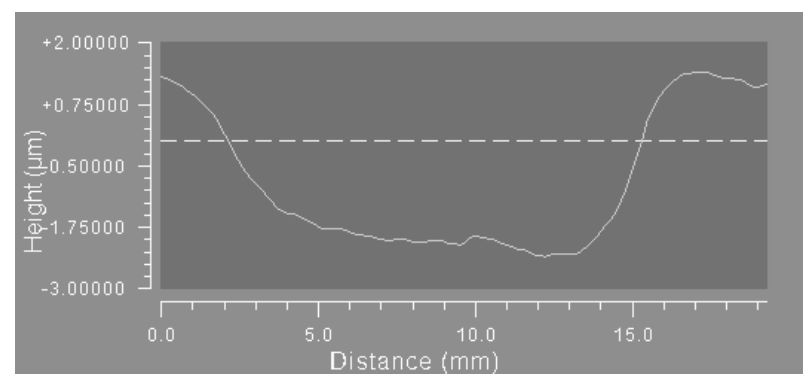

Fig. 4. Slightly worn tool on N-LAF21

\section{References}

[1] M. Schinhaerl a. o., Precision Engineering, VOL. 32, pages 47-54, [2008],

\footnotetext{
* Corresponding author: christian.vogt $@$ th-deg.de
} 